\title{
Genetic variants in insulin-like growth factor binding protein- 3 are associated with prostate cancer susceptibility in Eastern Chinese Han men
}

This article was published in the following Dove Press journal:

OncoTargets and Therapy

22 December 2015

Number of times this article has been viewed

\author{
Guiming Zhang ${ }^{1-3}$ \\ Yao Zhu', \\ Fang Liu ${ }^{4,5}$ \\ Chengyuan $\mathrm{Gu}^{1,2}$ \\ Haitao Chen ${ }^{4,5}$ \\ Jianfeng $\mathrm{Xu}^{4-6}$ \\ Dingwei $\mathrm{Ye}^{1,2}$
}

'Department of Urology, Fudan University Shanghai Cancer Center, ${ }^{2}$ Department of Oncology, Shanghai Medical College, Fudan University, Shanghai, ${ }^{3}$ Department of Urology, The Affiliated Hospital of Qingdao University, Shandong, ${ }^{4}$ Fudan Institute of Urology, Huashan Hospital, Fudan University, ${ }^{5}$ State Key Laboratory of Genetic Engineering, School of Life Sciences, Fudan University, Shanghai, People's Republic of China; ${ }^{6}$ Center for Cancer Genomics, Wake Forest School of Medicine, Winston-Salem, NC, USA
Correspondence: Dingwei Ye Department of Urology, Fudan University Shanghai Cancer Center, No 270, Dong'an Road, Shanghai, People's Republic of China Tel +86 2l 64175590

Email yedingweil963@I26.com
Background: Growing evidence has indicated that insulin-like growth factor binding protein-3 (IGFBP-3) polymorphisms are associated with altered risk of prostate cancer (PCa). However, few studies have been conducted in Chinese population to validate this association.

Materials and methods: Herein, we examined the association between genetic variants in the IGFBP-3 gene and PCa risk in the Chinese Han population based on a genome-wide association study (1,417 cases and 1,008 controls), and replicated three genetic variants loci in an independent case-control study (1,755 cases and 1,523 controls) using Sequenom platform. Logistic regression analyses were performed to estimate odds ratios (ORs) and $95 \%$ confidence intervals (95\% CIs).

Results: We found that in the discovery stage, rs 9691259 (OR $=0.691,95 \%$ CI: $0.587-0.814$, $P<0.001)$ and $\mathrm{rs} 6950179$ (OR $=1.420,95 \% \mathrm{CI}: 1.201-1.677, P<0.001)$ were significantly associated with PCa risk, whereas rs 2854744 showed a marginal association with PCa risk. In the replication stage, the association between rs9691259 and rs6950179 and PCa risk was not replicated, whereas rs 2854744 conferred a significant association with $\mathrm{PCa}$ risk $(\mathrm{OR}=1.399$, 95\% CI: $1.010-1.937, P=0.043)$. After combining the two stages, we found that rs9691259, rs6950179, and rs2854744 were all significantly associated with PCa risk.

Conclusion: This study suggests that IGFBP-3 genetic variants are significantly associated with PCa risk in the Chinese population.

Keywords: IGFBP-3, polymorphism, case-control study, genetic susceptibility

\section{Introduction}

Over the past few decades, much attention has been paid to the important role of metabolic syndrome (MS) in carcinogenesis. As a key factor of MS, insulin resistant denotes an interrupted state in the biological response to insulin. Under these circumstances, disordered expression and/or function of the insulin-like growth factor (IGF) axis is considered to be involved in the development and progression of various cancers, including prostate cancer (PCa). IGF binding protein-3 (IGFBP-3) is an important member of the IGFBP family, which binds more than $90 \%$ of circulating IGF-1 with high affinity and specificity, and regulates its biological activities. In vitro studies have demonstrated that IGFBP-3 independently inhibits cell proliferation and induces apoptosis of PCa cells. ${ }^{1,2}$ In addition, IGFBP-3 can exert an inhibitory role in PCa cell adhesion and motility. ${ }^{3}$ Moreover, using IGFBP-3 knockout mice, Mehta et al revealed that IGFBP-3 can suppress distant PCa metastases. ${ }^{4}$

Although PCa incidence is significantly lower in the People's Republic of China than in Western countries, disease-related morbidity is increasing year by year according 
to recent epidemiological studies. ${ }^{5}$ Genetic influence that explains $57 \%$ of all PCa based on the results of a twin study is considered vital; ${ }^{6}$ however, PCa etiology is still not well determined. Some rare germline mutations, such as HOXB13, were demonstrated to be closely associated with PCa risk in Chinese men, ${ }^{7}$ whereas germline sequence variants of some other important genes participating in PCa development, PTEN for instance, were not found to be intimately linked with PCa susceptibility. ${ }^{8}$ Likewise, the impact of genetic variants in IGFBP-3 on PCa risk varies significantly. Hernandez et al reported a two-fold increase in PCa risk for African-American men homozygous for the IGFBP-3 -202 C allele (rs2854744). ${ }^{9}$ Another study group observed no significant associations between IGFBP-3 rs2854744 and PCa risk in both AfricanAmericans and Caucasians. ${ }^{10}$ These inconsistent results may embody the influence of different ethnic backgrounds and environmental factors. Furthermore, most of these studies are limited to developed countries or a few single-nucleotide polymorphisms (SNPs), and no study to date has been conducted to examine the relationship between IGFBP-3 genetic variants and $\mathrm{PCa}$ risk in the Chinese population. Therefore, more polymorphic sites from a large-scale population are important to better understand the role of IGFBP-3 SNPs in PCa carcinogenesis. In the present study, we performed, for the first time in Southeastern Chinese men, a two-stage casecontrol study to evaluate whether IGFBP-3 polymorphisms are associated with $\mathrm{PCa}$ risk.

\section{Materials and methods Study subjects}

The present case-control study included two stages: data from a genome-wide association studies (GWAS) stage and a replication stage. The recruitment of study subjects in the GWAS stage has been described previously. ${ }^{11}$ Briefly, there were 1,417 hospital-based cases with pathologically confirmed PCa and 1,008 cancer-free controls that came from four districts and counties in the Shanghai, People's Republic of China area in the discovery stage. The replication stage included 1,755 pathologically confirmed $\mathrm{PCa}$ cases and 1,523 controls that visited for routine physical examination in local hospitals in Shanghai, Nanjing, and surrounding areas. All subjects were unrelated Han Chinese, and cases and controls were not overlapped in the two stages. Blood samples were obtained from each participant for DNA extraction once consent was acquired. Essential information, such as age, prostate-specific antigen (PSA) levels, history of cancer, as well as pathological data of PCa, was collected from standardized questionnaires and medical records. The study protocols were approved by the Institutional Research Review Boards of Fudan University Shanghai Cancer Center, Huashan Hospital, Fudan University, and other participating institutions, and written informed consent was obtained from all subjects before participation.

\section{SNP genotyping}

All SNPs in the IGFBP-3 gene were selected from the International HapMap Project database (http://hapmap.ncbi.

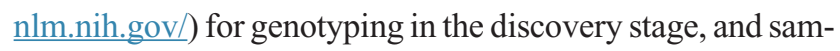
ples were genotyped on the MassARRAY iPLEX (Sequenom Inc., San Diego, CA, USA) platform. Standard quality control procedures to filter out unqualified DNA samples and SNPs were performed as described previously.Untyped SNPs were input according to reference data from the 1,000 Genomes Project CHB + JPT subjects by the IMPUTE2 program. ${ }^{12}$ Based on the following criteria, qualified SNPs were filtered as follows: 1) imputation quality score $>0.8 ; 2$ ) call rate $>95 \%$; 3) minor allele frequency $\geq 5 \%$ in Chinese Han populations; and 4) $P$-value for Hardy-Weinberg equilibrium in controls $>0.001$. In the replication stage, genotyping was performed using the Sequenom platform, and allelic discrimination was analyzed by the SDS v 2.4 (Applied Biosystems, Inc., Carlsbad, CA, USA) software. Two negative controls were included in each reaction plate, and genotyping was randomly repeated in $10 \%$ of samples, achieving a $100 \%$ concordance for all samples. Technicians who performed the genotyping procedures were blinded to the status of all subjects.

\section{Statistical methods}

Pearson's chi-squared tests were performed to analyze the agreement with Hardy-Weinberg equilibrium among the controls. Unconditional logistic regression analysis was used to estimate odds ratios (ORs) and 95\% confidence intervals (CIs) for PCa risk with adjustment for age. All statistical tests were two-sided, and carried out by the SAS software (version 9.2; SAS Institute Inc., Cary, NC, USA).

\section{Results}

The clinicopathological characteristics of all subjects are listed in Table 1. There was no significant difference in age between cases and controls, in either the discovery stage or the replication stage. As expected, PCa patients presented with much higher PSA levels compared with controls. In both stages, the Gleason scores of patients were mostly $\geq 7$.

We used data from the GWAS stage where a total of 690 SNPs in the IGFBP-3 gene were detected. Nine SNPs (rs9691259, rs6964415, rs7793447, rs17172664, rs6976880, 
Table I Clinicopathological characteristics of subjects in the two stages

\begin{tabular}{|c|c|c|c|c|}
\hline \multirow[t]{3}{*}{ Variables } & \multicolumn{2}{|c|}{ Discovery stage } & \multicolumn{2}{|c|}{ Replication stage } \\
\hline & Case & Control & Case & Control \\
\hline & $(n=1,4 \mid 7)$ & $(n=I, 008)$ & $(n=l, 755)$ & $(n=I, 523)$ \\
\hline $\mathrm{Age}^{\mathrm{a}}($ mean $\pm S D)$ & $71.3 \pm 8.1$ & $62.1 \pm 10.0$ & $70.1 \pm 7.7$ & $67.9 \pm 6.3$ \\
\hline \multicolumn{5}{|l|}{ PSA levels ${ }^{\mathrm{b}}(\mathrm{ng} / \mathrm{mL})$} \\
\hline$\leq 4.0$ & $55(3.9)$ & 965 (95.8) & $32(1.8)$ & I,373 (90.2) \\
\hline$>4.0,<10.0$ & $186(13.1)$ & $32(3.2)$ & $275(15.6)$ & II 8 (7.7) \\
\hline$\geq 10.0$ & I,098 (77.5) & $9(0.9)$ & $968(55.2)$ & $32(2.1)$ \\
\hline Missing & $78(5.5)$ & $2(0.1)$ & $480(27.4)$ & $0(0)$ \\
\hline \multicolumn{5}{|l|}{ T stage } \\
\hline $\mathrm{TI}$ & $180(12.7)$ & NA & $79(4.5)$ & NA \\
\hline $\mathrm{T} 2$ & $547(38.6)$ & NA & $412(23.5)$ & NA \\
\hline T3 & $359(25.3)$ & NA & $224(12.8)$ & NA \\
\hline $\mathrm{T} 4$ & 137 (9.7) & NA & $186(10.6)$ & NA \\
\hline Missing & $194(13.7)$ & NA & $854(48.6)$ & NA \\
\hline \multicolumn{5}{|l|}{$N$ stage } \\
\hline No & $786(55.5)$ & NA & $740(42.2)$ & NA \\
\hline $\mathrm{NI}$ & $369(26.0)$ & NA & 144 (8.2) & NA \\
\hline $\mathrm{Nx}$ & $262(18.5)$ & NA & 871 (49.6) & NA \\
\hline \multicolumn{5}{|l|}{ M stage } \\
\hline Mo & $832(58.7)$ & NA & $680(38.8)$ & NA \\
\hline MI & $440(31.1)$ & NA & $234(13.3)$ & NA \\
\hline$M x$ & $145(10.2)$ & NA & $84 \mid(47.9)$ & NA \\
\hline \multicolumn{5}{|l|}{ Gleason score } \\
\hline$<7$ & $355(25.1)$ & NA & $243(13.8)$ & NA \\
\hline$\geq 7$ & $993(70.1)$ & NA & $895(51.0)$ & NA \\
\hline Missing & $69(4.8)$ & NA & $617(35.2)$ & NA \\
\hline
\end{tabular}

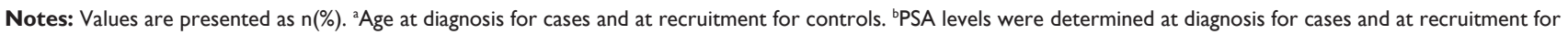
controls.

Abbreviations: NA, not available; PSA, prostate-specific antigen; SD, standard deviation.

rs6950179, rs1077750, rs7794166, and rs6970565) achieved a statistical level of $10^{-5}$. Among these nine SNPs, rs6964415, rs7793447, rs17172664, and rs6976880 were in strong linkage disequilibrium (LD; $r^{2}>0.8$ ) with rs9691259, whereas rs1077750, rs7794166, and rs6970565 were in strong LD $\left(r^{2}>0.8\right)$ with rs6950179. Among the two groups, rs9691259 and rs6950179 reached the highest statistical level, respectively. Hence, we selected these two SNPs for further validation. In addition, rs 2854744 indicated a marginal association with PCa risk $(P=0.052)$. Because quite a few studies to date have reported that rs 2854744 is associated with the risk of developing many cancers, ${ }^{9,13-15}$ including $\mathrm{PCa}$, we included this SNP in our replication stage.

Detailed information about the relationship between the three SNPs and PCa risk is indicated in Table 2. In the discovery stage, the $\mathrm{G}$ allele of rs 9691259 was associated with a decreased risk of $\mathrm{PCa}$, whereas the $\mathrm{C}$ allele of rs6950179 was associated with an increased PCa risk. The A allele of rs 2854744 conferred a marginal association with an increased PCa risk. In the replication stage, the association between rs9691259 and rs6950179 and PCa risk was not replicated.
However, patients carrying the homozygous mutant of rs2854744 presented with an increased PCa risk after adjustment for age (OR $=1.399,95 \%$ CI: $1.010-1.937, P=0.043$ ). A significant association was also observed in the dominant genetic model regarding rs2854744 and PCa risk $(\mathrm{OR}=1.168$, 95\% CI: $1.013-1.347, P=0.032)$. After combining data from the two stages, rs9691259, rs6950179, and rs2854744 were all significantly associated with PCa risk (Table 2).

\section{Discussion}

In the present two-stage case-control study, we found that three IGFBP-3 genetic variants (rs9691259, rs6950179, and rs2854744) were significantly associated with PCa risk. To the best of our knowledge, this is the first report of an association between IGFBP-3 polymorphisms and PCa risk in the Chinese Han population.

The incidence of MS is increasing worldwide, and MS-related cancers, including $\mathrm{PCa}$, have become a public health issue. Lund Haheim et al conducted a large-scale research project in Scandinavia and reported that MS was positively associated with PCa risk. ${ }^{16}$ Another study found 


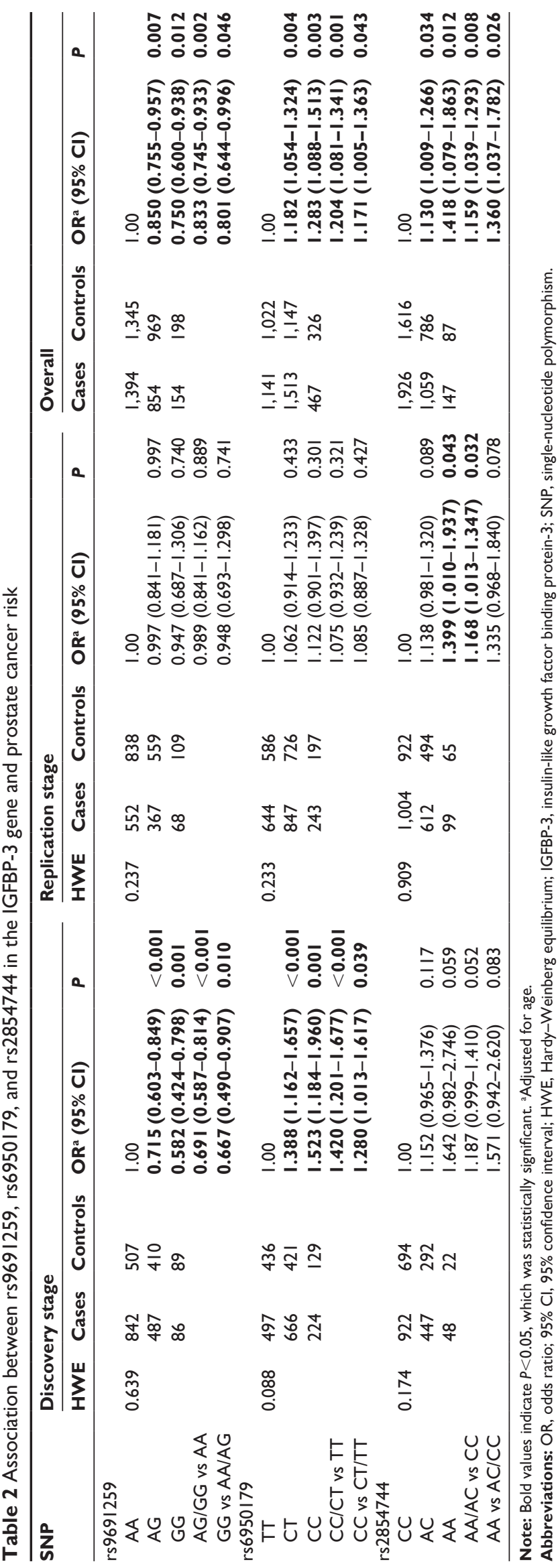

that MS was closely related with PCa progression. ${ }^{17}$ As the key mechanism responsible for MS, IGFs and IGFBPs are thought to be important in $\mathrm{PCa}$ carcinogenesis. However, despite the inhibitory role of IGFBP-3 in vitro in PCa development, conflicting conclusions have been reported regarding the relationship between serum IGFBP-3 levels and PCa risk, as well as IGFBP-3 polymorphisms and $\mathrm{PCa}$ risk. The association between decreased serum IGFBP-3 levels and increased PCa risk has been reported, ${ }^{18,19}$ whereas some studies did not observe an influence of IGFBP-3 levels on PCa risk. ${ }^{20,21}$ In a multi-ethnic large-scale study including 2,320 PCa cases and 2,290 controls, Cheng et al found that common genetic variation in the IGFBP-3 gene did not substantially influence PCa susceptibility. ${ }^{13}$ Similar results were also reported by other studies, although some found a significant association between IGFBP-3 genetic variants and serum IGFBP-3 concentration. ${ }^{10,15}$ However, significant relationship between IGFBP-3 polymorphisms and PCa risk has been reported by many studies. ${ }^{9} 18$ Likewise, our study observed a positive association between IGFBP-3 genetic variants and PCa risk. Furthermore, Hoyo et al found that the association between IGFBP-3 genetic variants and $\mathrm{PCa}$ susceptibility was only significant in those with Gleason score $<7$ rather than $\geq 7$, in both white and black men. ${ }^{22}$ There are several explanations for these inconsistent results. As we know, genetic susceptibility may differ according to ethnic background, and natural selection can result in genetic diversity that might be sensitive to local environments. In addition, many factors, such as insufficient statistical power, differences in study population characteristics, allelic heterogeneity, allele frequency and/or LD differences across various races, population stratification, differences in effect sizes, and gene-environment interactions, may contribute to the lack of replication of genetic associations or the observation of different relationships. ${ }^{23}$ Therefore, further studies combining more ethnic backgrounds are necessary to validate our observations.

Previous studies regarding the association between IGFBP-3 genetic variants and cancer mainly focused on a specific SNP, rs2854744, which locates at position -202 in the promoter region of IGFBP-3 gene. This A/C polymorphism has been reported to be highly correlated to circulating IGFBP-3 levels, and mutant $\mathrm{C}$ allele is associated with decreased IGFBP-3 levels. ${ }^{14,24}$ This may explain in part the underlying mechanisms of the impact of genetic variants on clinical phenotype. Unfortunately, serum IGFBP-3 levels were not determined in our study, and we could not assess whether IGFBP-3 polymorphisms contribute to circulating 
IGFBP-3 levels. However, the present study has several strengths. First, we performed our research with a relatively large number of samples in the People's Republic of China, which may enhance our statistical power. Second, we validated our observations in a replication stage based on a GWAS dataset. Compared with most previous studies that examined limited SNPs, our GWAS stage covered a large number of genetic variants. For example, for the first time, we found a significant association between rs9691259 and rs6950179 and PCa risk. Third, we validated the promising associations in the discovery stage in an independent cohort. This replication design likely makes our findings more conclusive.

Despite these strengths, our study still has some limitations. First, our study was conducted retrospectively, which carries an intrinsic bias. Second, our discovery stage study was performed using MassARRAY iPLEX platform, which might represent an incomplete coverage on genetic variants in the IGFBP-3 gene. Although additional imputation analyses may improve the study effectiveness, the possibility that some independent SNPs may be associated with PCa risk could not be excluded. Third, some information was inadequate, especially in the replication stage. For example, PSA levels and Gleason score were missing in many PCa patients. In addition, some other clinical data that might influence PCa risk, such as body mass index and history of diabetes, were not gathered in our database. All this missing information may lead to the deficiency of adjustment of potential confounding factors, as well as further stratification analyses, which might weaken our conclusion to a certain extent. Hence, our study requires further validation from well-designed prospective studies with adequate clinicopathological data.

\section{Conclusion}

The current two-stage case-control study found that rs9691259, rs6950179, and rs2854744 in the IGFBP-3 gene were significantly associated with altered PCa risk in the Chinese Han population. Our study adds more evidence to the current knowledge about the relationship between IGFBP-3 polymorphisms and cancer risk, and further highlights the important role of IGFBP-3 in PCa carcinogenesis.

\section{Acknowledgments}

This study was supported in part by a grant from the National Natural Science Foundation of China (No 81272837) and the National Natural Science Foundation of Shandong Province (Grant No ZR2009CM140). We thank Edanz Group Ltd. for the language polishing.

\section{Disclosure}

The authors report no conflicts of interest in this work.

\section{References}

1. Silha JV, Sheppard PC, Mishra S, et al. Insulin-like growth factor (IGF) binding protein-3 attenuates prostate tumor growth by IGFdependent and IGF-independent mechanisms. Endocrinology. 2006; 147(5):2112-2121.

2. Liu B, Lee KW, Anzo M, et al. Insulin-like growth factor-binding protein-3 inhibition of prostate cancer growth involves suppression of angiogenesis. Oncogene. 2007;26(12):1811-1819.

3. Massoner P, Colleselli D, Matscheski A, et al. Novel mechanism of IGF-binding protein-3 action on prostate cancer cells: inhibition of proliferation, adhesion, and motility. Endocr Relat Cancer. 2009;16(3): 795-808.

4. Mehta HH, Gao Q, Galet C, et al. IGFBP-3 is a metastasis suppression gene in prostate cancer. Cancer Res. 2011;71(15):5154-5163.

5. He J, Chen WQ. Chinese Cancer Registry Annual Report. Beijing: Military Medical Science Press; 2012: P29.

6. Page WF, Braun MM, Partin AW, Caporaso N, Walsh P. Heredity and prostate cancer: a study of World War II veteran twins. Prostate. 1997; 33(4):240-245.

7. Lin X, Qu L, Chen Z, et al. A novel germline mutation in HOXB13 is associated with prostate cancer risk in Chinese men. Prostate. 2013; 73(2):169-175.

8. Xie CC, Lu L, Sun J, et al. Germ-line sequence variants of PTEN do not have an important role in hereditary and non-hereditary prostate cancer susceptibility. J Hum Genet. 2011;56(7):496-502.

9. Hernandez W, Grenade C, Santos ER, et al. IGF-1 and IGFBP-3 gene variants influence on serum levels and prostate cancer risk in AfricanAmericans. Carcinogenesis. 2007;28(10):2154-2159.

10. Schildkraut JM, Demark-Wahnefried W, Wenham RM, et al. IGF1 (CA)19 repeat and IGFBP3 -202 A/C genotypes and the risk of prostate cancer in Black and White men. Cancer Epidemiol Biomarkers Prev. 2005; 14(2):403-408.

11. Chen $Y$, Xin X, Li J, et al. RTK/ERK pathway under natural selection associated with prostate cancer. PLoS One. 2013;8(11):e78254.

12. Xu J, Mo Z, Ye D, et al. Genome-wide association study in Chinese men identifies two new prostate cancer risk loci at $9 \mathrm{q} 31.2$ and $19 \mathrm{q} 13.4$. Nat Genet. 2012;44(11):1231-1235.

13. Cheng I, Penney KL, Stram DO, et al. Haplotype-based association studies of IGFBP1 and IGFBP3 with prostate and breast cancer risk: the multiethnic cohort. Cancer Epidemiol Biomarkers Prev. 2006; 15(10):1993-1997.

14. Deal C, Ma J, Wilkin F, et al. Novel promoter polymorphism in insulinlike growth factor-binding protein-3: correlation with serum levels and interaction with known regulators. J Clin Endocrinol Metab. 2001; 86(3):1274-1280.

15. Friedrichsen DM, Hawley S, Shu J, et al. IGF-I and IGFBP-3 polymorphisms and risk of prostate cancer. Prostate. 2005;65(1):44-51.

16. Lund Haheim L, Wisloff TF, Holme I, Nafstad P. Metabolic syndrome predicts prostate cancer in a cohort of middle-aged Norwegian men followed for 27 years. Am J Epidemiol. 2006;164(8):769-774.

17. Zhang GM, Zhu Y, Dong DH, et al. The association between metabolic syndrome and advanced prostate cancer in Chinese patients receiving radical prostatectomy. Asian J Androl. 2015;17(5): 839-844.

18. Park K, Kim JH, Jeon HG, Byun SS, Lee E. Influence of IGFBP3 gene polymorphisms on IGFBP3 serum levels and the risk of prostate cancer in low-risk Korean men. Urology. 2010;75(6):1516.e1-e7.

19. Safarinejad MR, Shafiei N, Safarinejad S. Relationship of insulin-like growth factor (IGF) binding protein-3 (IGFBP-3) gene polymorphism with the susceptibility to development of prostate cancer and influence on serum levels of IGF-I, and IGFBP-3. Growth Horm IGF Res. 2011;21(3):146-154. 
20. Janssen JA, Wildhagen MF, Ito $\mathrm{K}$, et al. Circulating free insulin-like growth factor (IGF)-I, total IGF-I, and IGF binding protein-3 levels do not predict the future risk to develop prostate cancer: results of a case-control study involving 201 patients within a population-based screening with a 4-year interval. J Clin Endocrinol Metab. 2004;89(9): 4391-4396.

21. Morris JK, George LM, Wu T, Wald NJ. Insulin-like growth factors and cancer: no role in screening. Evidence from the BUPA study and metaanalysis of prospective epidemiological studies. Br J Cancer. 2006; 95(1):112-117.

22. Hoyo C, Grubber J, Demark-Wahnefried W, et al. Grade-specific prostate cancer associations of IGF1 (CA)19 repeats and IGFBP3-202A/C in blacks and whites. J Natl Med Assoc. 2007;99(7):718-722.
23. Bryant EK, Dressen AS, Bunker $\mathrm{CH}$, et al. A multiethnic replication study of plasma lipoprotein levels-associated SNPs identified in recent GWAS. PLoS One. 2013;8(5):e63469.

24. Jernstrom H, Deal C, Wilkin F, et al. Genetic and nongenetic factors associated with variation of plasma levels of insulin-like growth factor-I and insulin-like growth factor-binding protein-3 in healthy premenopausal women. Cancer Epidemiol Biomarkers Prev. 2001;10(4): 377-384.

\section{Publish your work in this journal}

OncoTargets and Therapy is an international, peer-reviewed, open access journal focusing on the pathological basis of all cancers, potential targets for therapy and treatment protocols employed to improve the management of cancer patients. The journal also focuses on the impact of management programs and new therapeutic agents and protocols on

\section{Dovepress}

patient perspectives such as quality of life, adherence and satisfaction. The manuscript management system is completely online and includes a very quick and fair peer-review system, which is all easy to use. Visit http://www.dovepress.com/testimonials.php to read real quotes from published authors.

Submit your manuscript here: http://www.dovepress.com/oncotargets-and-therapy-journal 